\title{
LOCATION TRACKING IN WIRELESS LOCAL AREA NETWORKS WITH ADAPTIVE RADIO MAPS
}

\author{
A. Kushki, K.N. Plataniotis, A.N. Venetsanopoulos \\ \{azadeh, kostas,anv\}@dsp.utoronto.ca \\ Multimedia Laboratory, The Edward S. Rogers Sr. Dept. of Electrical \& Computer Engineering \\ University of Toronto
}

\begin{abstract}
This paper proposes a dynamic MMSE estimator for tracking mobile users in indoor Wireless Local Area Networks (WLAN) based on Received Signal Strength (RSS). The method uses a training-based static estimate obtained by an adaptive kernel density estimator as the input into a Kalman Filter. Predictions from the filter are used during the next iteration to adaptively select a subset of training data, contained in a radio map, for the static estimator. Experimental results show that the combination of the Kalman filter and the adaptive radio map technique results in nearly $0.5 \mathrm{~m}(20 \%)$ improvement in Root Mean Square location accuracy when compared to static localization.
\end{abstract}

\section{INTRODUCTION}

Location-aware mobile devices have become an amenity since the introduction of the E-911 mandate by the U.S. Federal Communications Commission, requiring wireless carriers to provide location information on emergency callers. Cellular network infrastructures have subsequently served as milieux for development of locationbased services. Location-awareness has also proved propitious in indoor applications such as location-based network access and security, automatic resource assignment, guidance of persons with disabilities, monitoring of patients, and location-sensitive information delivery. Unfortunately, the level of localization accuracy needed in such indoor applications cannot be achieved by the existing cellularbased methods. As an alternative, Wireless Local Networks (WLAN) can be used for indoor localization as they offer ubiquitous coverage in large areas. Furthermore, Network Interface Cards require no additional hardware for reporting the Received Signal Strength (RSS) which is used for localization of mobile clients. Lastly, because localization is carried out on the mobile client, no invasive sensing, processing, and central storage is performed.

Despite the advantages of WLAN localization systems, the dependence relationship between RSS and physical locations cannot be trivially determined. Severe multipath and shadowing conditions and non-line-of-sight propagation caused by the presence of walls, humans, and other rigid objects give rise to a complex and timevarying radio environment, making the estimation problem particularly challenging.

Existing WLAN localization works can be divided into two categories namely, static and dynamic. Static methods [1,2] use only power measurements at the given time step to formulate the location estimate. The estimation procedure generally involves an offline

This work is partially supported by the Natural Sciences \& Engineering Research Council of Canada. phase in which survey data measurements are collected at known locations in the environment. The set of these survey locations together with their corresponding RSS measurements comprise what is known as a radio map. Readings from the mobile user (MU) during online operation are then compared against this radio map and the corresponding estimate is returned. Existing works generally rely on nearest neighbour classifiers or Bayesian estimator (Maximum Likelihood/Maximum A Posteriori) to obtain the location estimate from the radio map data.

Considering that motion of human beings is generally correlated overtime, dynamic localization solutions exploit past location estimates, in addition to current RSS measurements, to calculate the location estimate. The dynamic solution may entail a running average of previous estimates [2] or rely on more sophisticated methods such as the Kalman filter [3] or Markov-model based solutions [4,5].

Traditionally, dynamic localization solutions have aimed to improve the current estimate based on the users' previous locations. This paper goes a step further and exploits information regarding future locations of the MU in forming the current estimate. Specifically, predictions from the dynamic estimator are used to select a subset of the radio map used for localization. We show how this spatial filtering of the radio map can result in improved location estimates as compared with a nonadaptive technique by restricting the estimate to relevant regions of the environment.

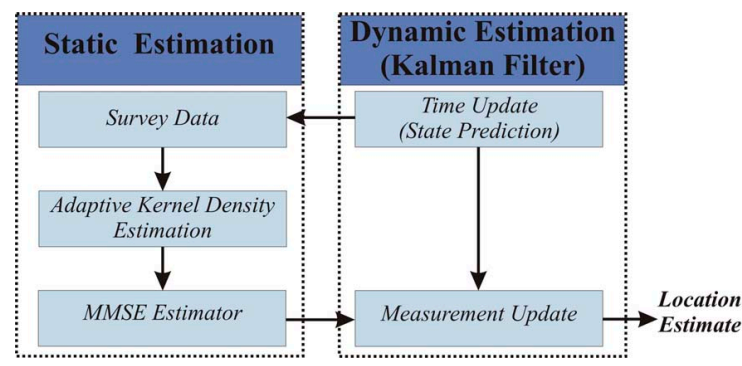

Fig. 1. Overview of the proposed system.

The outline of the proposed method is shown in Fig.1. We first employ an Minimum Mean Square Error (MMSE) estimator for static localization as described in Section 2. This estimate is used as the measurement input into a dynamic estimator (the Kalman filter, in this case). Location predictions from the filter are sent back to the static estimator to adaptively select a subset of the training data for the next estimation step. Details of the dynamic tracking and radio map filtering are discussed in Section 3 and experimental results are presented and discussed in Section 4. 


\section{STATIC MMSE LOCALIZATION}

This section describes a static estimator that uses RSS measurements at time step $k$ to generate a location estimate at this time using the radio map constructed in an offline stage. The radio map contains a set of RSS measurements for $n$ locations in the environment, designated as survey points. Denote this map as $\mathcal{S}=$ $\left\{\left\{\mathbf{p}_{1}, \mathbf{r}_{1,1}, \ldots, \mathbf{r}_{1, T_{1}}\right\}, \ldots,\left\{\mathbf{p}_{n}, \mathbf{r}_{n, 1}, \ldots, \mathbf{r}_{n, T_{n}}\right\}\right\}$ where, $\mathbf{r}_{i, j}$ is a vector of readings from $m$ access points at time step $j$, for survey location $\mathbf{p}_{i}$.

Since the location of a mobile user is represented in the Cartesian space, an appropriate estimation criterion is the minimization of the squared error between a location estimate and its true position. This view leads to a Minimum Mean Square Error (MMSE) estimator for localization which corresponds to the conditional expectation given below:

$$
\hat{\mathbf{p}}_{M M S E}=E(\mathbf{p} \mid \mathbf{r})=\frac{\int_{\mathcal{P}} \mathbf{p} f(\mathbf{r} \mid \mathbf{p}) f(\mathbf{p}) d \mathbf{p}}{\int_{\mathcal{P}} f(\mathbf{r} \mid \mathbf{p}) f(\mathbf{p}) d \mathbf{p}},
$$

where $\mathcal{P}$ denotes the region of space for which the conditional density $f(\mathbf{p} \mid \mathbf{r})$ is non-zero. The densities $f(\mathbf{p})$ and $f(\mathbf{r} \mid \mathbf{p})$ are unknown, they are estimated from the radio map data using nonparametric techniques as described below.

The density $f(\mathbf{p})$ contains the prior knowledge regarding the position of the mobile user before making an RSS observation. Generally, a uniform prior density can be assumed over the entire physical space. Such an approach, however, ignores the physical topology of the environment in terms of walls, furniture, and other spaces that cannot be occupied by the user. This work exploits knowledge of the location of survey points $\left\{\mathbf{p}_{1}, \ldots, \mathbf{p}_{n}\right\}$ and considers these as samples of the prior density. Then, the empirical probability density function (epdf) [6] is used as the estimate of the prior density as shown below.

$$
\hat{f}(\mathbf{p})=\frac{1}{n} \sum_{\mathbf{p}_{i} \in \mathcal{S}} \delta\left(\mathbf{p}-\mathbf{p}_{i}\right) .
$$

Substitution of(2) in (1) gives the estimate as a weighted sum of the survey points:

$$
\hat{\mathbf{p}}_{M M S E} \approx=\frac{\sum_{\mathbf{p}_{i} \in \mathcal{S}} \mathbf{p}_{i} f\left(\mathbf{r} \mid \mathbf{p}_{i}\right)}{\sum_{\mathbf{p}_{i} \in \mathcal{S}} f\left(\mathbf{r} \mid \mathbf{p}_{i}\right)}
$$

Next, the estimation of the density $f\left(\mathbf{r} \mid \mathbf{p}_{i}\right)$ is considered. This density is reported to be heavily dependent on environmental conditions and may possibly be Gaussian, multimodal, or asymmetric and long-tailed. In light of this, nonparametric tools are chosen to estimate this density as they operate solely on the structure present in the survey data and require no prior assumptions on the form of the density. As an alternative to the histogram estimate commonly used in prior work, this work makes use of a kernel density estimator (KDE) due to its superior theoretical properties such as integrated bias and variance $[6,7]$.

$$
\hat{f}\left(\mathbf{r} \mid \mathbf{p}_{i}\right)=\frac{1}{T_{i}} \sum_{t=1}^{T_{i}} h_{\mathbf{r}}^{-d} K\left(\frac{\mathbf{r}-\mathbf{r}_{i, t}}{h_{\mathbf{r}}}\right) .
$$

In (4), $d$ is the dimension of $\mathbf{r}, T_{i}$ is the number of time samples collected at $\mathbf{p}_{i}, K(\cdot)$ is a zero-mean, non-negative kernel function with unit area (a Gaussian kernel is used in this work). The parameter $h$ is the kernel width used to control the region of influence of each survey value on the final estimate. Large smoothing parameters capture the global structure of the density whereas smaller values result in increased details. Ideally, the bandwidth should be small in areas where the density is large and large where density is small, specially in long-tail distributions that require sufficient smoothing in the tail. Because RSS distributions are reported to be asymmetric and long-tailed in many situations, an adaptive kernel density estimator (AKDE) [6] is used to provide variable smoothing as shown in (5).

$$
\hat{f}\left(\mathbf{r} \mid \mathbf{p}_{i}\right)=\frac{1}{T_{i}} \sum_{t=1}^{T_{i}} h_{\mathbf{r}}^{-d} \lambda_{i, t}^{-d} K\left(\frac{\mathbf{r}-\mathbf{r}_{i, t}}{h_{\mathbf{r}} \lambda_{i, t}}\right),
$$

where $\lambda_{i, t}=\left(\check{f}\left(\mathbf{r}_{i, t}\right)\right)^{-0.5}$ are local bandwidth multipliers obtained from a pilot density estimate $\check{f}\left(\mathbf{r}_{i, t}\right)$ (e.g. constant bandwidth estimator of (4)). Note that these values only depend on the survey data and can therefore be calculated offline. As a final modification, storage requirements of (5) can be reduced by grouping temporal samples with equal values. Denoting the temporal frequency of sample $\mathbf{r}_{i, t}$ as $n_{t}$, the estimator becomes:

$$
\hat{f}\left(\mathbf{r} \mid \mathbf{p}_{i}\right)=\frac{1}{T_{i}} \sum_{t=1}^{T_{i}^{\prime}} n_{t} h_{\mathbf{r}}^{-d} \lambda_{i, t}^{-d} K\left(\frac{\mathbf{r}-\mathbf{r}_{i, t}}{h_{\mathbf{r}} \lambda_{i, t}}\right) .
$$

\section{ADAPTIVE TRACKING}

The static estimator of the previous section uses RSS observations from time step $k$ and the entire radio map information to form the location estimate $\hat{\mathbf{p}}(k)$ at this time step. Fortunately, human motion is not completely random and the locations of the mobile user are generally correlated overtime. Thus, the estimator can also benefit from the knowledge of past location estimates in addition to the RSS measurements. Another consequence of the time correlation of locations is that future locations of the MU can be predicted from past estimates. We propose to exploit this information by adaptively selecting relevant regions of the radio map to be included during estimation. The details of the proposed dynamic tracker and the adaptive feature selection method are discussed in the rest of this section.

\subsection{Dynamic Tracking}

This work uses a Kalman filter (KF) for dynamic estimation. The Kalman filter is a model-based recursive MMSE estimator of the state of a linear time-varying system with noisy observations. The state vector in our case contains the Cartesian coordinates of the user as well as his/her velocity and is denoted as $\mathbf{p}=\left[p_{x}, v_{x}, p_{y}, v_{y}\right]^{T}$. The outputs of the filter at time step $k$ depend on measurements up to time $k$ and are the state estimate, $\hat{\mathbf{p}}_{K F}(k \mid k)$, and the error covariance associated with this estimate, $\hat{P}_{K F}(k \mid k)$.

To form the state estimate, the Kalman filter relies on two models. The first model describes the evolution of the state over time whereas the second relates the measurements to the state. In our case, the system equation corresponds to a model for motion of pedestrians. The measurement equation must relate the RSS measurements to the position of the user. However, since the relationship between RSS and physical locations is non-trivial in indoor spaces, we use a preprocessor to generate pseudo-measurements for the filter in a manner similar to $[3,8]$. As shown in Fig.1, this preprocessor corresponds to the static estimator proposed previously. The output of the static estimator, which corresponds to a location estimate based on RSS measurements, is used as the measurement input for 
the filter. This measurement is then combined with previous location estimates by the KF to produce an improved estimate. The system and measurement equations described herein are as follows:

$$
\begin{aligned}
\mathbf{p}(k+1) & =F \mathbf{p}(k)+w(k), \\
\hat{\mathbf{p}}_{M M S E}(k) & =H \mathbf{p}(k)+v(k),
\end{aligned}
$$

where $\mathbf{p}(\mathrm{k})$ is true location of the user at time step $k, \hat{\mathbf{p}}_{M M S E}(k)$ is the estimate produced by the static estimator at time $k, w(k) \sim$ $\mathcal{N}(0, Q), v(k) \sim \mathcal{N}(0, R(k))$ are uncorrelated, zero-mean, white, and Gaussian and $F$ and $H$ are the system transition and measurement matrices given the sampling period $T$ :

$$
F=\left(\begin{array}{llll}
1 & T & 0 & 0 \\
0 & 1 & 0 & 0 \\
0 & 0 & 1 & T \\
0 & 0 & 0 & 1
\end{array}\right) \quad H=\left(\begin{array}{llll}
1 & 0 & 0 & 0 \\
0 & 0 & 1 & 0
\end{array}\right)
$$

The measurement noise covariance can be directly obtained from the static estimator [8]:

$$
\begin{aligned}
R(k) & =\operatorname{Cov}\left(\hat{\mathbf{p}}_{M M S E} \mid \mathbf{r}(k)\right) \\
& \approx \sum_{i=1}^{n} \mathbf{p}_{i} \mathbf{p}_{i}^{T} \hat{f}\left(\mathbf{r} \mid \mathbf{p}_{i}\right)-\hat{\mathbf{p}}_{M M S E} \hat{\mathbf{p}}_{M M S E}^{T}
\end{aligned}
$$

Although the Kalman filter is an optimum MMSE estimator for a linear-Gaussian system, it produces a suboptimal result in the above scenario. This is mainly due to the violation of assumptions on the measurement noise. Specifically, the residuals from the static estimator may not be zero-mean, white and Gaussian in practise. Despite this, however, Section 4 shows that the above modelling assumptions do produce acceptable results.

The Kalman filter calculates the final location estimate in a two step process. First, the state equation is used to predict the next state of the system. Second, the incoming measurements are used to adjust this prediction and produce the final estimate. Denoting the estimate of the state at time $k$ given measurements up to time $j$ as $\hat{\mathbf{p}}(k \mid j)$ and the error covariance as $\hat{P}_{K F}(k \mid j)$, the two steps are summarized below.

$$
\begin{aligned}
& \text { 1. Prediction: } \\
& \hat{\mathbf{p}}_{K F}(k \mid k) \rightarrow \hat{\mathbf{p}}_{K F}(k+1 \mid k) \\
& \hat{P}_{K F}(k \mid k) \rightarrow \hat{P}_{K F}(k+1 \mid k)
\end{aligned}
$$

2. Update

$$
\begin{aligned}
& \hat{\mathbf{p}}_{K F}(k+1 \mid k) \rightarrow \hat{\mathbf{p}}_{K F}(k+1 \mid k+1) \\
& \hat{P}_{K F}(k+1 \mid k) \rightarrow \hat{P}_{K F}(k+1 \mid k+1)
\end{aligned}
$$

\subsection{Adaptive Feature Selection}

With reference to Fig.1, the dynamic estimator (KF) uses estimates from the static estimator during its update step. The static estimator, in turn, relies on the survey data of the entire radio map to produce its estimate. Note, however, that the relationship between the observed RSS and spatial positions is a many-to-many one. This is partially due to the time-varying nature of RSS at fixed locations. In other words, the same RSS can be observed at different locations from the same access point depending on the geometry of the space and time of observation. In order to resolve such ambiguities, this paper proposes an adaptive method for selection of the survey points used by the static estimator.
The adaptive selection method relies on the predication from the Kalman filter to select relevant survey points at each step. To do this, recall that the confidence region for the estimate $\hat{\mathbf{p}}_{K F}$, assuming the positioning error is approximately Gaussian, is determined by the " $g$-sigma" ellipsoid defined as [9]:

$$
\left(\mathbf{p}-\hat{\mathbf{p}}_{K F}\right)^{T} \hat{P}_{K F}^{-1}\left(\mathbf{p}-\hat{\mathbf{p}}_{K F}\right)=g^{2} .
$$

The semi-axes of the above ellipsoid are $g$ times the square root of the eigenvalues of the covariance matrix $\hat{P}_{K F}$. The desired probability concentration can be achieved by selecting the parameter $g$. For example, the probability of being inside the 3 -sigma ellipse is $98.9 \%$.

In the proposed method, the survey points falling into the $g$ sigma ellipse are sent back to the static estimator. This feedback results in the inclusion of survey points that are physically "close" the predicted location estimate. As previously mentioned, this spatial filtering operator has the effect of reducing the number of outliers during RSS comparisons and restricting the static estimator to the relevant region of the environment. Experimental results reported in the next section show the effectiveness of the proposed method in reducing the positioning error.

\section{EXPERIMENTS \& RESULTS}

This section investigates the effect of the proposed adaptive radio map technique on positioning accuracy. To show this, we consider the performance in 4 cases. Namely, the final location estimate is taken to be the output of: 1) the static estimator ${ }^{1}$ 2) the Kalman filter using the static estimator as the preprocessor 3) the static estimator that uses adaptive radio maps 4) the Kalman filter using the static, adaptive estimator as the preprocessor.

Positioning accuracy of the methods under consideration is quantified using the Root Mean Square Error (RMSE) measure where positioning error is calculated as the Euclidean distance between the true position and its estimate in the Cartesian plane.

\subsection{Experimental Setup}

The performance comparisons are done using data obtained from an office environment on the forth floor of an eight story building at the University of Toronto. Fig.2 shows the experimentation area layout and the location of survey points, depicted as black circles.

Although there were several access points (AP) spread throughout the floor, the reported results, use only four APs at each point. The measurements were made on an Toshiba Tablet PC with a Pentium III processor, a Netgear wireless card, and Windows XP operating system. RSS measurements were obtained by a publicly available network sniffer software, NetStumbler ${ }^{2}$. A total of 150 RSS sample were collected at the sampling rate of 1 over two intervals in two different days. Measurements were collected for a total of 39 survey points, covering a laboratory and a hallway as shown in Fig.2. The points were separated by 2 meters on a uniform grid where not restricted by physical constraints. For testing purposes, RSS samples were collected over 14 tracks of varying length. In addition, the track data was collected at two constant speeds of $.5 \mathrm{~m} / \mathrm{s}$ and $.3 \mathrm{~m} / \mathrm{s}$ using

\footnotetext{
${ }^{1}$ Preliminary results comparing the static estimator to state of the art methods can be found in [7]. These results show that the proposed static estimator is in fact superior to its nearest neighbor and maximum a posteriori counterparts. In this light and due to space limitations, we refrain from presenting further results on this topic.

${ }^{2} \mathrm{http}: / /$ www.netstumbler.org
} 


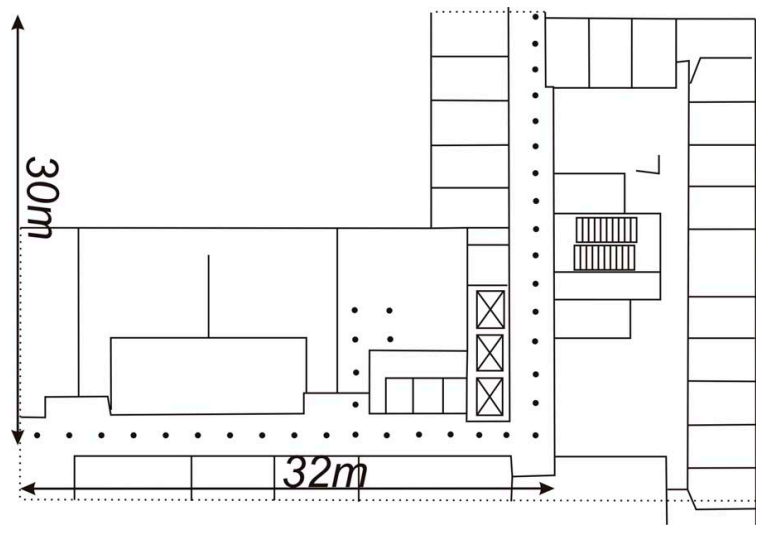

Fig. 2. Map of the experimentation environment.

an ER1 robot. The orientation of the robot was kept the same during training and testing to eliminate orientation effects on the results.

\subsection{Results}

Table 1 reports the RMSE accuracy of the four methods using the 3 -sigma ellipse in the adaptive cases. It can be seen that the adaptive radio map technique does in fact improve the positioning accuracy. As previously mentioned, this is due to the spatial filtering of survey point information and elimination of outliers in this set. By using location predications as an additional feature, the proposed adaptive method is able to resolve ambiguities caused by the many-to-many RSS-position relation in the RSS space.

Table 1. RMSE results using the 3-sigma ellipse.

\begin{tabular}{|l|c|c|}
\hline & fixed survey points & adaptive points \\
\hline \hline Static localization & $2.14 \mathrm{~m}$ & $1.73 \mathrm{~m}$ \\
\hline Dynamic localization & $1.92 \mathrm{~m}$ & $1.67 \mathrm{~m}$ \\
\hline
\end{tabular}

We next consider the effect of the parameter $g$ on the performance of the adaptive methods. It is excepted that small values of $g$ degrade this performance as inaccuracies in the static preprocessor lead to exclusion of significant survey information. The erroneous estimates are then sent back to the estimator and coupled with the small confidence region cause further deviation from the relevant portion of the radio map. Indeed, the RMSE results depicted in Fig. 3 demonstrate that for small $g$, the adaptive methods perform poorly.

For values of $g$ around 3, the adaptive methods show significant improvement over the other four methods as an appropriate confidence interval results in selection of the correct portion of the radio map. As the value of $g$ increases and the confidence ellipse grows, the number of included survey points increases and the performance of the adaptive methods converge to that of nonadaptive techniques that include the entire radio map.

\section{CONCLUSION}

This paper has proposed a dynamic localization solution based on adaptive radio maps. Location predictions from a dynamic tracker are used to narrow down the positioning problem to a subset of possible locations in the environment. The results presented herein

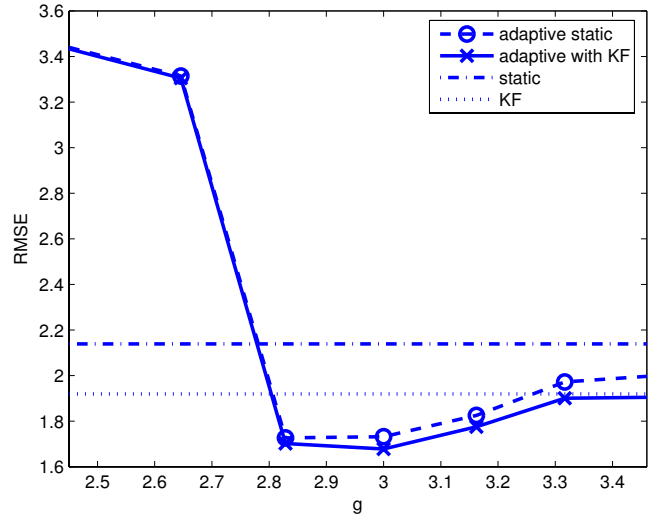

Fig. 3. Position accuracy for the four methods as a function of size of the confidence ellipse.

demonstrate that such a method can in fact lead to significant improvements in terms of positioning accuracy.

\section{REFERENCES}

[1] P. Bahl and V.N. Padmanabhan, "RADAR: an in-building RFbased user location and tracking system," in Proceedings of IEEE Infocom, 2000, vol. 2, pp. 775-784.

[2] Moustafa Yousief, Horus: A WLAN-Based Indoor Location Determination System, Ph.D. thesis, University of Maryland, 2004.

[3] I. Guvenc, C.T. Abdallah, R. Jordan, and O. Dedeoglu, "Enhancements to RSS based indoor tracking systems using kalman filters," in Proceedings of The International Signal Processing Conference and Global Signal Processing Expo, 2003.

[4] A.M. Ladd, K.E. Bekris, A.P. Rudys, D.S. Wallach, and L.E. Kavraki, "On the feasibility of using wireless ethernet for indoor localization," IEEE Transactions on Robotics and Automation, vol. 20, no. 3, pp. 555 - 559, 2004.

[5] C. Gentile and L. Klein-Berndt, "Robust location using system dynamics and motion constraints," in Proceedings of The IEEE International Conference on Communications, 2004, vol. 3, pp. 1360-1364.

[6] David W. Scott, Multivariate Density Estimation, John Wiley and Sons, 1992.

[7] A. Kushki, K.N. Plataniotis, A.N.Venetsanopoulos, and C. Regazzoni, "Radio map fusion for indoor positioning in wireless local area networks," in Proceedings of the Eighth International Conference on Information Fusion, 2005.

[8] M. McGuire and K.N. Plataniotis, "Dynamic model-based filtering for mobile terminal location estimation," IEEE Trans. on Vehicular Technology, vol. 52, no. 4, pp. 1012-1031, 2003.

[9] Yaakov Bar-Shalom, X.-Rong Li, and Thiagalingam Kirubarajan, Estimation with applications to tracking and navigation, New York : Wiley, 2001. 